\title{
TRAINING EFFECTS OF PLYOMETRIC TRAINING ON JUMP PARAMETERS IN D- AND D/C-SQUAD BADMINTON PLAYERS
}

\author{
M. Fröhlich ${ }^{1 *}$--- H. Felder ${ }^{2}$--- M. Reuter \\ 'Saarland University, Institute for Sport Science, Saarbruecken, Germany \\ ${ }^{\circ}$ Olympic Training Center Rhineland Palatinate/Saarland, Saarbruecken, Germany; Institute for Prevention and Public Health, \\ University of Applied Sciences, Saarbruecken, Germany \\ ${ }^{3}$ Saarland University, Institute for Sport Science, Saarbruecken, Germany; Institute for Prevention and Public Health, University of \\ Applied Sciences, Saarbruecken, Germany
}

\begin{abstract}
Plyometric training is popular among individuals involved in dynamic sports, and plyometric exercises such as jumping, hopping, skipping and bounding are executed with the goal of increasing dynamic muscular performance, especially jumping. Much less information is available on the effectiveness of plyometric training $(P T)$ in badminton, where jumping high (e.g. forehand overhead jump-smash) is important for success. The aim of the study was to investigate the effects of an 8-week periodized PT program on jumping high and power among male and female junior badminton players, using high-impact bilateral plyometric exercises. Starting and finishing with the biomechanical diagnostics of the squat jump (SJ), counter movement jump (CMJ), and drop jump (DJ) on force plates, kinematic analysis of forehand overhead smashes, anthropometric data as well as force data for pre-and posttest were analyzed. Before and after the biomechanical diagnostics, the players ( $n=11$ ) undergo an 8-week PT (2 units per week) with a total of 2286 jumps. 8 male and 3 female junior badminton players (age: 16.0 11.6 years, height: $175.5 \pm 9.9 \mathrm{~cm}$, mass: $69.3 \pm 11.4 \mathrm{~kg}$ ) were tested in jumping high and forehand overhead jump-smashes performance. Looking at the plyometric strength parameters of the squat jump $\left(P<0.05 ; d_{z}=0.8\right)$ and the floor reaction-time of the drop jump $\left(P<0.05 ; d_{z}=1.1\right)$, the positive effect of the 8-week PT in junior badminton players is significant. Consequently, this form of training is essential for the development of junior and top-level badminton players. Moreover, the study has shown that the contact of the overhead smash cannot be increased by improving plyometric strength training $(P>0.05)$. Therefore, the focus must be on technical training. Consequently, it is considered to be important to include short-term PT in in-season preparation in order to improve complex badminton-specific dynamic performance (smash-jumping).
\end{abstract}

Keywords: Plyometric training, Badminton players, Jumping high, Forehand overhead smash, Biomechanical analysis, Training preparation.

Received: 5 December 2014/ Revised: 23 December 2014/ Accepted: 27 December 2014/ Published: 31 December 2014 


\section{Contribution/ Originality}

The paper's primary contribution is finding that an 8-week plyometric training in junior badminton players can significantly improve different parameters in jumping high but the plyometric training has no direct influence of the overhead smash. Therefore, the focus must be on technical training.

\section{INTRODUCTION}

Besides table tennis and tennis, badminton is one of the fastest Olympic racket and net sports in the world. World-class athletes are able to achieve short-term, maximum shuttlecock speeds of $288 \mathrm{~km} / \mathrm{h}$ to $365 \mathrm{~km} / \mathrm{h}$ (Tsai and Chang, 1998). This competitive sport is also characterized by badminton-specific running paths, jumps, and lunges, as well as by the continuous change between accelerated and decelerated movements. During an entire match, a player covers a distance of approx. 1800-1900 m (Liddle et al., 1996). The average match and break time is approx. 6.4 seconds and 12.9 seconds (Table 1), and the average number of shots is 6.1 per rally (Cabello and González-Badillo, 2003).

Table-1. Badminton match characteristics (EPT=effective playing time)(Faude et al., 2007)

\begin{tabular}{l|l|l|l|l|l}
\hline $\begin{array}{l}\text { Rally } \\
\text { time (s) }\end{array}$ & $\begin{array}{l}\text { Rest } \\
\text { time (s) }\end{array}$ & $\begin{array}{l}\text { Work } \\
\text { density }\end{array}$ & $\begin{array}{l}\text { Number of } \\
\text { shots per rally }\end{array}$ & $\begin{array}{l}\text { Shots per } \\
\text { rally time }\left(\mathbf{s}^{-1}\right)\end{array}$ & $\begin{array}{l}\text { EPT } \\
(\%)\end{array}$ \\
\hline $5.5 \pm 4.0$ & $11.4 \pm 6.0$ & $\begin{array}{l}0.51 \pm 0.3 \\
4\end{array}$ & $5.1 \pm 3.9$ & $0.92 \pm 0.31$ & $31.2 \pm 2.8$ \\
\hline
\end{tabular}

The physical strain is of an interval type, which results in high demands of energy provision. Accordingly, a badminton player's lactate values are usually above the aerobic-anaerobic threshold for continuous strain with values of 3.8-4.7 mmol/1 (Majumdar et al., 1997; Cabello and González-Badillo, 2003). The corresponding heart rates values are specified at 80-95\% of the maximum heart rate (Liddle et al., 1996; Majumdar et al., 1997; Cabello and González-Badillo, 2003; Faude et al., 2007). Table 2 shows the physiological requirements for badminton.

Table-2. Physiological requirements profile in badminton

\begin{tabular}{|c|c|c|}
\hline Parameter & Result & Author(s) \\
\hline $\begin{array}{l}\text { Heart rate in percent of the } \\
\text { maximum heart rate }\end{array}$ & $\begin{array}{l}\text { Ø } 86 \% \mathrm{HR}_{\max } \\
\text { Ø } 93 \% \mathrm{HR}_{\max } \\
78.3-99.8 \% \mathrm{HR}_{\max }\end{array}$ & $\begin{array}{l}\text { (Majumdar et al., 1997) } \\
\text { (Liddle et al., 1996) } \\
\text { (Faude } \text { et al., 2007) }\end{array}$ \\
\hline $\begin{array}{l}\text { Lactate concentration during } \\
\text { a badminton match }\end{array}$ & $3.8-4.7 \mathrm{mmol} / 1$ & $\begin{array}{l}\text { (Cabello and González-Badillo, } \\
\text { 2003), (Majumdar et al., 1997) }\end{array}$ \\
\hline $\begin{array}{l}\mathrm{VO}_{2 \max } \text { in percent of the } \\
\text { maximum } \mathrm{VO}_{2 \max }\end{array}$ & $\begin{array}{l}\text { Ø } 73.7 \% \mathrm{VO}_{2 \max } \\
45.7-100.9 \% \mathrm{VO}_{2 \max }\end{array}$ & $\begin{array}{l}\text { (Cabello and González-Badillo, } \\
\text { 2003) }\end{array}$ \\
\hline
\end{tabular}

At the technical level of the badminton-specific requirements profile, the forehand overhead smash is most significant in terms of maximum shuttlecock speeds (Tang et al., 1995). With approx. $20 \%$ it is also the second-most frequent shot in badminton (Hong and Tong, 2000). The 
objective of the forehand overhead smash is a direct winner or the aggressive preparation for a winner. The higher the athlete hits the shuttlecock - i.e., indirectly, the higher the height of the jump - the steeper the trajectory and the shorter the path of the shuttlecock. This enables the player to optimize the utilization of the court size (Rambely et al., 2005).

The technical difficulty of the forehand overhead smash becomes apparent when observing the shot technique in detail. The height of the hitting point depends on various factors, such as jump ability, spatiotemporal perception, flight behavior of the incoming shuttlecock, as well as the temporal interconnection of sub-segments. The optimum hitting point is achieved when the entire body is stretched, which means an optimally coordinated stretching of the upper body and the playing arm. An even higher hitting point can be achieved when performing the shot as a jump smash, which requires optimum jumping power and jumping height (Tsai and Chang, 1998; Tsai et al., 2000). As effective as the forehand overhead smash may be in terms of a point-winning technique, this type of shot technique requires a high degree of specific technique mastering and coordinative spatiotemporal, dynamic precision (Hong, 1993). The technical complexity of the forehand overhead smash can be described as follows. To perform a forehand overhead smash, the player must go through various movement and orientation patterns. First, the player needs to analyze the shuttlecock trajectory in order to position the body optimally under the shuttlecock. Then, the player needs to select the optimal timing for the jump. During the jump, the racket must be positioned in the best position to build up the body tension required. If these factors are perfectly coordinated, a forehand overhead jump smash can be performed with a precise hitting point at the highest point possible.

Relative force is a major factor in achieving the highest possible shuttlecock hitting point. Therefore, maximum strength training, speed training, and various forms of plyometric training, such as jumps, jump sequences, and jump combinations show significant advantages over methods expanding the muscle cross section (Bobbert, 1990). Elastic and neurophysiologic (reactive) mechanisms can result in increased strength development.

The combination of eccentric and subsequent concentric muscle action represents a frequent movement pattern (e.g., a jump), also known as stretch shortening cycle (SSC). Contractions during the SSC generate a higher movement impulse in the concentric phase (take-off phase, in a narrower sense) than a purely concentric contraction. The reasons for this lie in the storage of elastic energy and the triggering of muscle stretching reflexes during the eccentric phase. The ability to realize a highly concentric strength impulse within the shortest time span possible from within an eccentric (decelerating) movement is called reactive movement behavior ("fast SSC" within 90-200 ms). Factors limiting or influencing factors include: elastic energy storage, shortrange elastic stiffness (SRES), interconnection time, stretch strain and stretch amplitude, stretch speed, and neuronal factors.

Numerous authors describe training effects in terms of jump height improvement through SSC training (reactive training, plyometric training) (Kyröläinen and Komi, 1994). Sialis (2004) provides a detailed and decided representation. In summary, it can be stated that training of this type of contraction can have considerable impact on both muscular-skeletal (Witzke and Snow, 
2000; Kato et al., 2006; Kubo et al., 2007; Wu et al., 2010) and neuromuscular adaptations (Kyröläinen et al., 2005; Malisoux et al., 2006; Grosset et al., 2009; Saez-Saez de Villarreal et al., 2010), as well as on jumping behavior, even if ambiguous statements stemming from detailed observations (exhaustion behavior, dosage behavior, pause design, etc.) exist (Sialis, 2004).

In general, two (match-based) objectives apply to badminton jump movements: achieving maximum height, and achieving a specific point with part of the body/equipment.

Both objectives are of utmost significance in badminton and are therefore to be taken into account in terms of practical training work and technique transfer. On the one hand, plyometric types of training can improve badminton-specific speed requirements and movement patterns, such as running and jump movements, lunges, steps, changes in direction, etc. (Joshi, 2012). On the other hand, at a muscle-physiological level, plyometric training exploits impulses of the preinnervation and reflex potentiation during the stretch shortening cycle as well as the elastic component of the muscle (Gehri et al., 1998), which, in turn leads to an improvement of jump and speed performance (Meylan and Malatesta, 2009; Kannas et al., 2012; Martinez-Lopez et al., 2012). Using plyometric training in badminton is now considered key to boosting sport-specific capacities (Sturgess and Newton, 2008) with concrete training recommendations (strain parameter) being only rare, however (Middleton et al., 2013).

Ultimately, it is assumed that plyometric training types result in higher take-offs and thus, implicitly, also in higher shot speeds - to minimize the time the shuttlecock spends in the air - for forehand overhead smashes. Based on these considerations, two questions should be examined:

1) Can 8-week plyometric training improve the jump performance of junior badminton players in the $\mathrm{D}$ - and $\mathrm{D} / \mathrm{C}$ squad?

2) Can 8-week plyometric training improve the hitting point in forehand overhead smashes of junior badminton players in the $\mathrm{D}$ - and $\mathrm{D} / \mathrm{C}$ squad?

\section{METHODS}

\subsection{Subjects}

A total of eleven members of the D- or D/C-squad of the Saarland and German badminton association participated voluntarily in the explorative, quasi-experimental intervention study including pre-post measurement. Due to the explorative, quasi-experimental character of the study with pre- and post-measurement, learning, adaptation, development, test and interaction effects cannot be excluded besides the assumed intervention effects. Furthermore, due to the small size of the sample, the natural group, and the non-withholding of treatment, an assignment to treatment and control groups or other treatment groups was not applied. The treatment was not administered in intervals so that dosage-effectiveness interconnections cannot be excluded, either.

The players were all under $15(\mathrm{n}=3)$ and under $19(\mathrm{n}=8)$ and had had no previous experience in systematic plyometric training. The average age of the eight boys and three girls was $16.0 \pm 1.6$ years with an average height of $175.5 \pm 9.9 \mathrm{~cm}$ and an average body weight of $69.3 \pm 11.4 \mathrm{~kg}$. Due to organizational reasons a control group was not implemented. 


\subsection{Operationalization of Jump Parameters}

To operationalize the vertical jump power or reactive force behavior, the performances in squat jump (SJ), counter-movement Jump (CMJ), and drop jump (DJ) were determined using a contact mat and micro controller. In all jump test forms, the hands were fixed at the hip during the entire jump. Each test person received technique instructions before testing started (jump demonstration and two familiarization test jumps). The individual jumps were performed in immediate succession with a one-minute break in between jump series. Subsequently, a 2-D video analysis (Utilius Easy Inspect) identified kinematic parameters of maximum jump height at the shuttlecock hitting point, and racket height during the forehand overhead smash. Technique instructions were given here, as well (jump demonstration and two test jumps). The forehand overhead smash was performed in intervals of 5 seconds. For all tests, the testers had the instruction of "maximum take-off". Figure 1 illustrates the individual kinematic parameters of the hitting technique for the forehand overhead smash.

A total of seven jumps were performed for all jump forms, with the best and poorest jump having been canceled so that in the end five jumps were included in the analysis (Faude et al., 2010). The arithmetic mean for pre- and post-test was then calculated based on these five jumps. To compare the DJ data for jump height and floor contact time the so-called landing parameter was calculated for economic reasons (drop height plus jump height divided by floor contact time).

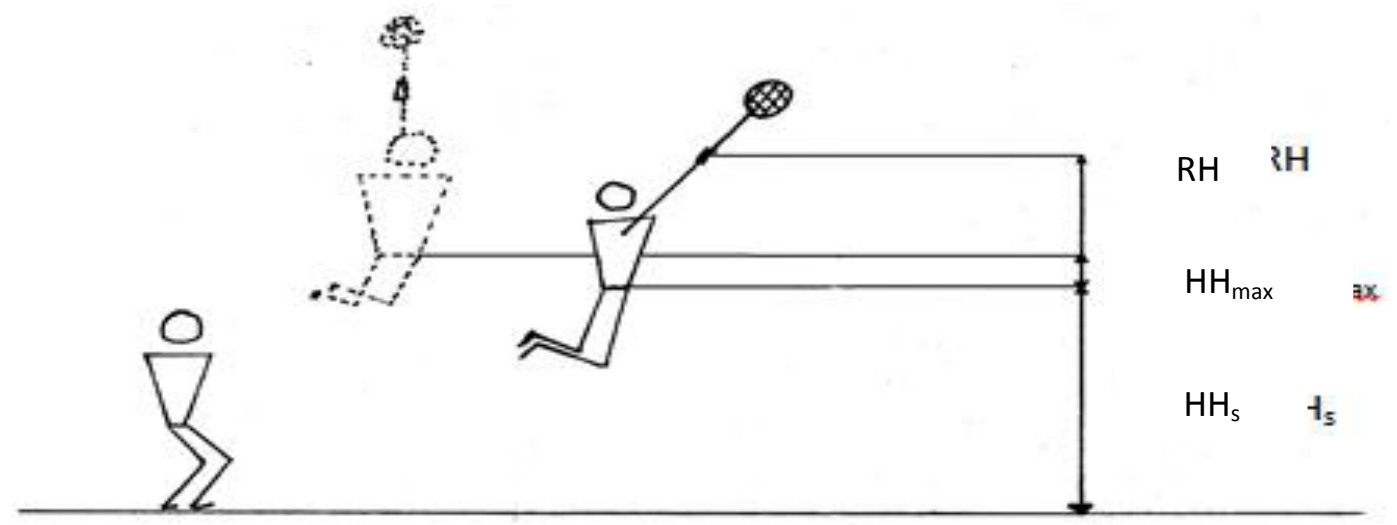

Figure-1. Kinematic jump and racket parameters of a forehand overhead smash at the optimal hitting point $(\mathrm{RH}=$ maximum racket height at shuttlecock hitting point, $\mathrm{HH}_{\max }=$ maximum hip height during the jump, $\mathrm{HH}_{\mathrm{s}}=$ jump height at shuttlecock hitting point)

\subsection{Treatment}

The plyometric training was performed during an intervention period of 8 weeks, twice a week. The duration of the individual training units was approximately 30 minutes and increased progressively over the number of jumps to be performed. The plyometric jump training took place before the actual badminton training. To avoid interaction effects between plyometric training and general badminton training endurance-specific strain was excluded. Nevertheless, interaction effects between jump training and general badminton training cannot be completely 
excluded. A general warm-up program was followed by 8 exercises (jumps, jump sequences, and jump combinations) for simple (jumps without additional load or equipment) and medium (jumps over obstacles) plyometrics one-legged or with both legs for SJ, CMJ, and DJ (Table 3). Jump series and pauses were kept constant and adjusted to the individual performance level. The exercise sequence was predefined and of a progressive character - the longer the training was performed, the more strain was added by eccentric and intensive-plyometric strain (bounces). The first week of training served as a familiarization phase with only 156 jumps to be carried out per training unit. To avoid physiological, biomechanical, and coordinative overload, DJs and mediumplyometric exercises were not performed in the first week of training. In the course of the weeks, the number of jumps was successively increased from 204 (second week) to 360 (seventh and eighth week). Thus, during the entire intervention phase, 2286 jumps had to be carried out.

Table-3. Plyometric training exercise overview

\begin{tabular}{|c|c|}
\hline Exercise & Description \\
\hline $\begin{array}{l}\text { 1. Squat jump (simple } \\
\text { plyometrics) }\end{array}$ & $\begin{array}{l}\text { Start position: } \\
\text { - Start position in squat position approx. } 90^{\circ} \\
\text { - Feet at shoulder width } \\
\text { - Hands placed on the hips } \\
\text { Motion: } \\
\text { - Maximum vertical take-off } \\
\text { - Direct upward movement by stretching the legs } \\
\text { - Landing position identical with take-off position }\end{array}$ \\
\hline $\begin{array}{l}\text { 2. Counter movement } \\
\text { jump (simple } \\
\text { plyometrics) }\end{array}$ & $\begin{array}{l}\text { Start position: } \\
\text { - Upright standing position } \\
\text { - Feet at shoulder width } \\
\text { - Hands placed on the hips } \\
\text { Motion: } \\
\text { - Swing movement (knees flexed at approx. } 90^{\circ} \text { ) } \\
\text { - Eccentric initial movement followed by maximum take-off } \\
\text { - Landing position identical with take-off position }\end{array}$ \\
\hline $\begin{array}{l}\text { 3. Drop jump (medium } \\
\text { plyometrics) }\end{array}$ & $\begin{array}{l}\text { Start position: } \\
\text { - Standing on vaulting box }(32 \mathrm{~cm}) \\
\text { - Hands placed on the hips } \\
\text { Motion: } \\
\text { - One-legged step forward from the vaulting box } \\
\text { - Landing with both legs with maximum reactive take-off up }\end{array}$ \\
\hline \multirow[t]{2}{*}{$\begin{array}{l}\text { 4. Triple jump with } \\
\text { both legs (simple } \\
\text { plyometrics) }\end{array}$} & $\begin{array}{l}\text { Start position: } \\
\text { - Upright standing position } \\
\text { - Feet at shoulder width } \\
\text { - Arms are swinging } \\
\text { Motion: } \\
\text { - Swing movement (knees flexed at approx. } 90^{\circ} \text { ) } \\
\text { - Maximum take-off forward-and-up with subsequent take- } \\
\quad \text { off }\end{array}$ \\
\hline & $\begin{array}{l}\text { Start position: } \\
\text { • Upright standing position on one leg }\end{array}$ \\
\hline
\end{tabular}




\begin{tabular}{|c|c|}
\hline $\begin{array}{l}\text { 5. One-legged triple } \\
\text { jump (simple } \\
\text { plyometrics) }\end{array}$ & $\begin{array}{l}\text { - Hands are swinging } \\
\text { Motion: } \\
\text { - Swing movement (knees flexed at approx. } 130^{\circ} \text { ) } \\
\text { - Maximum take-off from the ankle joint forward-and-up } \\
\text { with subsequent one-legged (same leg) take-off } \\
\text { Variation: } \\
\text { - Use obstacles }\end{array}$ \\
\hline $\begin{array}{l}\text { 6. Jump with both legs } \\
\text { over the obstacle(s) } \\
\text { (medium } \\
\text { plyometrics) }\end{array}$ & $\begin{array}{l}\text { Obstacles: } \\
\text { - Front obstacle } 15 \mathrm{~cm} \text {, rear obstacle } 30 \mathrm{~cm} \\
\text { Start position: } \\
\text { - Upright standing position } \\
\text { - Feet at shoulder width } \\
\text { - Arms are swinging } \\
\text { Motion: } \\
\text { - Swing movement (knee flexed at approx. } 90^{\circ} \text { ) } \\
\text { - Take-off forward-and-up with subsequent explosive take- } \\
\text { Variation: } \\
\text { - Place obstacles further apart }\end{array}$ \\
\hline $\begin{array}{l}\text { 7. Gym bench jumps } \\
\text { (medium } \\
\text { plyometrics) }\end{array}$ & $\begin{array}{l}\text { Start position: } \\
\text { - Upright standing position on gym bench } \\
\text { - Feet at shoulder width } \\
\text { - Hands placed at the hip } \\
\text { Motion: } \\
\text { - Drop jump from one bench to the next }\end{array}$ \\
\hline $\begin{array}{l}\text { 8. Gym bench-obstacle } \\
\text { jumps (medium } \\
\text { plyometrics) }\end{array}$ & $\begin{array}{l}\text { Start position: } \\
\text { - Upright standing position on gym bench } \\
\text { - Feet at shoulder width } \\
\text { - Hands placed at the hip } \\
\text { Motion: } \\
\text { - Combination of drop jump and obstacle jump with both } \\
\quad \text { legs (obstacle } 30 \mathrm{~cm})\end{array}$ \\
\hline
\end{tabular}

\subsection{Statistics}

The descriptive statistical analysis included factors such as the mean value, standard deviation, minimum and maximum values, as well as percentage and absolute changes. The interference-statistical calculation of significance was done using $\mathrm{T}$ test for paired samples. Precondition tests were carried out applying the usual methods (KS test for Gaussian distribution, Levené test for homogeneity of variance). The effect size $d_{z}$ was calculated as the

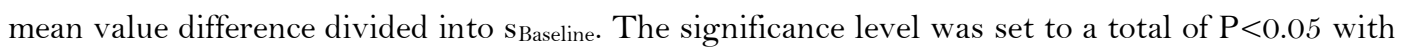
the Bonferroni correction $\left(\alpha^{\prime}=\alpha / \mathrm{m}\right)$ applied.

\section{RESULTS}

The 8-week plyometric training significantly increased the squat jump performance by 4.4 $\mathrm{cm}$ from an average $32.9 \pm 5.4 \mathrm{~cm}$ to $37.3 \pm 6.2 \mathrm{~cm}\left(\mathrm{P}<0.05 ; \mathrm{d}_{\mathrm{z}}=0.8\right)$. The average performance increase was at 13.7 percent (0.6\% minimum and $26.1 \%$ maximum). 
For the CMJ, the performance improvement was at an average of $6.0 \%$ ( $2 \mathrm{~cm}$ absolute). However, individual performances showed a broad range with values between $-7.2 \%$ and $+14.8 \%$. Overall, a considerable increase $\left(P>0.05 ; \mathrm{d}_{\mathrm{z}}=0.3\right)$ could not be identified between preand post-test from an average of $40.7 \pm 7.6 \mathrm{~cm}$ to $43.2 \pm 7.5 \mathrm{~cm}$.

For the DJ, as a measure for the reactive force performance, a significant improvement of the landing parameter from $3.9 \pm 0.4$ to $4.4 \pm 0.6$ was demonstrated. The average improvement was $12.1 \%$ with a range between $1.1 \%$ and $24.2 \%\left(\mathrm{P}<0.05 ; \mathrm{d}_{\mathrm{z}}=1.1\right)$.

The values identified using videographic means for maximum jump height $\left(\mathrm{HH}_{\max }\right)$, jump height at shuttlecock hitting point $\left(\mathrm{HH}_{\mathrm{s}}\right)$, and racket height $(\mathrm{RH})$ during the forehand overhead smash are shown in table 4 . The maximum jump heights increased considerably between pre- and post-test $\left(P<0.05 ; d_{z}=0.3\right)$, while the jump height at shuttlecock hitting point $\left(P>0.05 ; d_{z}=0.2\right)$ and the maximum racket height at shuttlecock hitting point $\left(\mathrm{P}>0.05 ; \mathrm{d}_{\mathrm{z}}=0.1\right)$ did not show reveal any significant changes. Absolute jump height improvements were measured at $4.4 \mathrm{~cm}\left(\mathrm{HH}_{\max }\right)$, $3.3 \mathrm{~cm}\left(\mathrm{HH}_{\mathrm{s}}\right)$, and $2.8 \mathrm{~cm}(\mathrm{RH})$.

Table-4. $2 \mathrm{D}$ video analysis results of the pre- and post-test

\begin{tabular}{|c|c|c|c|c|c|c|c|c|c|c|c|c|c|}
\hline VP & 1 & 2 & 3 & 4 & 5 & 6 & 7 & 8 & 9 & 10 & 11 & MW & SD \\
\hline $\mathrm{HH}_{\max }-\mathrm{E}$ & 131.4 & 170.4 & 149.6 & 152.2 & 128.4 & 151.2 & 146.8 & 166.2 & 175.6 & 149.2 & 165.8 & 153.3 & 15.1 \\
\hline $\mathrm{HH}_{\max }-\mathrm{A}$ & 139.6 & 177.4 & 152.8 & 158.8 & 130.8 & 155.6 & 147.8 & 174.8 & 172.4 & 154.6 & 170.4 & 157.7 & 15.0 \\
\hline $\mathrm{HH}_{\mathrm{s}}-\mathrm{E}$ & 130.4 & 165.4 & 147.6 & 147.2 & 127.4 & 150.6 & 144.4 & 158.8 & 173.6 & 146.2 & 163.0 & 150.4 & 14.1 \\
\hline $\mathrm{HH}_{\mathrm{s}}-\mathrm{A}$ & 138.2 & 173.6 & 150.4 & 152.8 & 129.8 & 146.2 & 146.2 & 168.0 & 169.2 & 150.2 & 166.4 & 153.7 & 14.0 \\
\hline RH-E & 248.0 & 291.6 & 252.2 & 276.2 & 230.2 & 249.8 & 249.0 & 274.6 & 298.2 & 256.4 & 265.8 & 262.9 & 20.5 \\
\hline RH-A & 260.6 & 300.2 & 256.8 & 281.2 & 230.6 & 252.2 & 250.2 & 289.6 & 285.0 & 254.6 & 262.2 & 265.7 & 20.7 \\
\hline
\end{tabular}

$\mathrm{HH}_{\max }=$ maximum hip height during jump in $\mathrm{cm}, \mathrm{HH}_{\mathrm{s}}=$ jump height at shuttlecock hitting point in $\mathrm{cm}, \mathrm{RH}=$ maximum racket height at shuttlecock hitting point in $\mathrm{cm}, \mathrm{E}=$ pre-test, $\mathrm{A}=$ post-test

\section{DISCUSSION AND PERSPECTIVE}

The results of this study point to the conclusion that additional plyometric training can positively influence the performance of junior athletes of the $\mathrm{D}$ - and $\mathrm{C} / \mathrm{D}$ badminton squad in terms of various jump parameters, such as squat jump and drop jump (Sialis, 2004; Markovic, 2007; Kannas et al., 2012). The positive performance improvement in squat jumps can be explained by the selection of individual exercises of simple and medium plyometrics within the training units. Since most exercises, jumps, or jump sequences of the training intervention did not require any swing movement - the actual jump was executed mostly from a concentric movement with a knee angle of more than 90 degrees - a high degree of congruence between dynamic training exercise and test modality can be assumed for the SJ. Due to the relatively low drop heights short floor contact times can be assumed, which in turn, leads to the assumption of a high degree of congruence between test and training requirements for the DJ. In contrast, no 
significant improvement was found for the CMJ based on the selected treatment - dynamic jumps mostly without swing movement and in the SSC. Overall, the varied jump training resulted in a performance increase of an average of $13.7 \%$ for SJ, $6.0 \%$ for CMJ, and $12.1 \%$ for DJ, although individual test persons did exhibit performance degradation. This can be explained inter alia by development and learning-based influences, sports-specific training age, neuronal and tendomuscular values, interaction with other training content of regular badminton training, etc.

When considering the videographically analyzed $\mathrm{HH}_{\max }, \mathrm{HH}_{\mathrm{s}}$, and $\mathrm{RH}$ parameters, the 8week training resulted in a significant increase (jump power) only for the maximum jump height (with the hip being the reference point). The two parameters jump height at shuttlecock hitting point and maximum racket height at shuttlecock hitting point - both representing a highly coordinative and spatiotemporal challenge - do not differ majorly in pre-test and post-test results. Thus, it could be concluded that the plyometric training applied improved jump power or jump height, while its influence on the technical component of the forehand overhead smash in the sense of a spatiotemporal improvement is rather negligible. This would mean that the influence of the optimal technique applied to the forehand overhead smash in the sense of a suitable combination of sub-segments outweighs pure jump force improvement. Nevertheless, this study does not serve any estimation to what extent the technical implementation may represent a corresponding limitation within the group examined.

\section{CONCLUSIONS PERTAINING TO PRACTICAL TRAINING}

The analysis showed that an 8-week, systematic, progressively increasing plyometric training can improve the jumping power of junior athletes of the D- and D/C squad (Kannas et al., 2012). Even though maximum jump height are not explicitly required in performance-oriented badminton, there are still implicit influences of jumping power and jump height on badmintonspecific hitting techniques, such as smashes and on badminton-specific running techniques, such as short, quick steps that mainly put strain on the forefoot. It is presumed that plyometric training content can positively influence the badminton-specific court speed (for example, by reducing floor contact time), which in turn has positive effects on competitive performance.

An opportune point in time for additional plyometric training for performance-oriented badminton players would be the preparation phase, which is usually scheduled in May and August. During this time, the physical foundation is established for the entire competitive sports season that runs from August to April including several seasonal peaks. Using plyometric training after a hypertrophy training block for the lower extremities is conceivable, with the training completed one to two weeks before the competitions start so that the players recover in time for the competitive phase.

Moreover, plyometric training should be based on individual preconditions. It is essential to ensure that the extent of stimulation and the training scope do not result in overstraining the tendomuscular structures. Since the extent of stimulation or strain intensity in plyometric training is decisively determined by drop height and predefined jump height in jump forms and combinations, it is important to make sure that, for example, the heel does not just yet touch the 
floor during a drop jump. The training scope can be intensified progressively with the number of jumps in line with performance increase. In principle, increasing the number of jumps is recommended to be implemented as a means of progression before increasing the drop height. However, it is important to assure that the complexities of plyometric training match the overall movement requirements of badminton and that no negative interaction effects with regular badminton training occur. Because of the high amount of mechanical and neuromuscular strain/fatigue, which can lead to an increased deterioration of cellular structures, regeneration is of key importance (Macaluso et al., 2012). To minimize the risk of injury by plyometric training, warm-up training is recommended to prepare the athlete in terms of coordination and muscle prestress for the challenges ahead. Rests are of major significance here. A rest of between one and two minutes is suggested. Also, reactive jumps on the court floor are recommended because on the one hand, this surface provides adequate buffer for the joints, and on the other hand, it is sufficiently hard for not reducing the stretch strain. Frequent pre-, intermediate, and post-tests as well as continuous training controls should be included in planning and executing a plyometric training in order to be able to evaluate the quality of the training program. Suitable reliable and valid test procedures are available: squat jump, counter movement jump, and drop jump, all performed on a contact mat.

\section{Funding: This study received no specific financial support.}

Competing Interests: The authors declare that they have no competing interests.

Contributors/Acknowledgement: All authors contributed equally to the conception and design of the study. We are grateful to Badminton World Federation (BWF) for the financial support of this study.

\section{REFERENCES}

Bobbert, M.F., 1990. Drop jumping as a training method for jumping ability. Sports Medizin, 9(1): 7-22.

Cabello, M.D. and J.J. González-Badillo, 2003. Analysis of the characteristics of competitive badminton. British Journal of Sports Medicine, 37(1): 62-66.

Faude, O., T. Meyer, F. Rosenberger, M. Fries, G. Huber and W. Kindermann, 2007. Physiological characteristics of badminton match play. European Journal of Applied Physiology, 100(4): 479-485.

Faude, O., A. Schlumberger, T. Fritsche, G. Treff and T. Meyer, 2010. Leistungsdiagnos tische testverfahren im fußball - methodische standards. Deutsche Zeitschrift für Sportmedizin, 61(6): 129-133.

Gehri, D.J., R. M., D. Kleiner and D. Kirkendall, 1998. A comparison of plyometric training techniques for improving vertical jump ability and energy production. Journal of Strength and Conditioning Research, 12(2): 85-89.

Grosset, J.F., J. Piscione, D. Lambertz and C. Perot, 2009. Paired changes in electromechanical delay and musculo-tendinous stiffness after endurance or plyometric training. European Journal of Applied Physiology, 105(1): 131-139.

Hong, Y., 1993. The biomechanics of badminton smash technique. In Proceedings XIV Symposium of Biomechanics in Sports. Paris: Société De Biomecanique: pp: 588-589. 
Hong, Y. and M. Tong, 2000. The playing pattern of the world's top single badminton players in competition. Journal of Human Movement Studies, 38: 185-200.

Joshi, I., 2012. Comparative analysis of 4 weeks agility training program and plyometric training on agility in badminton players - a randomised clinical trial. Dissertation. Kle University.

Kannas, T.M., E. Kellis and I.G. Amiridis, 2012. Incline plyometrics-induced improvement of jumping performance. European Journal of Applied Physiology, 112(6): 2353-2361.

Kato, T., T. Terashima, T. Yamashita, Y. Hatanaka, A. Honda and Y. Umemura, 2006. Effect of lowrepetition jump training on bone mineral density in young women. Journal Applied Physiology, $100(3): 839-843$.

Kubo, K., M. Morimoto, T. Komuro, H. Yata, N. Tsunoda, H. Kanehisa and T. Fukunaga, 2007. Effects of plyometric and weight training on muscle-tendon complex and jump performance. Medicine and Science in Sports and Exercise, 39(10): 1801-1810.

Kyröläinen, H., J. Avela, J.M. McBride, S. Koskinen, A.J. L., S. Sipila, T.E. Takala and P.V. Komi, 2005. Effects of power training on muscle structure and neuromuscular performance. Scandinavian Journal of Medicine and Science in Sports, 15(1): 58-64.

Kyröläinen, H. and P.V. Komi, 1994. Neuromuscular performance of lower limbs during voluntary and reflex activity in power- and endurance-trained athletes. European Journal of Applied Physiology, 69(3): 233-239.

Liddle, S.D., M.H. Murphy and W. Bleakley, 1996. A comparison of the physiological demands of singles and doubles badminton: A heart rate and time/motion analysis. Journal of Human Movement Studies, 30: 159-176.

Macaluso, F., A.W. Isaacs and K.H. Myburgh, 2012. Preferential type ii muscle fiber damage from plyometric exercise. Journal of Athletic Training, 47(4): 414-420.

Majumdar, P., G.L. Khanna, V. Malik, S. Sachdeva, M. Arif and M. Mandal, 1997. Physiological analysis to quantify training load in badminton. British Journal of Sports Medicine, $31(4)$ : 342- 345.

Malisoux, L., M. Francaux, H. Nielens, P. Renard, J. Lebacq and D. Theisen, 2006. Calcium sensitivity of human single muscle fibers following plyometric training. Medicine and Science in Sports and Exercise, 38(11): 1901-1908.

Markovic, G., 2007. Does plyometric training improve vertical jump height? A meta-analytical review. British Journal of Sports Medicine, 41(6): 349-355.

Martinez-Lopez, M.J., L. Benito-Martinez, F. Hita-Contreras, A. Lara-Sanchez and A. Martinez-Amat, 2012. Effects of electrostimulation and plyometric training program combination on jump height in teenage athletes. Journal of Sports Science and Medicine, 11(4): 727-735.

Meylan, C. and D. Malatesta, 2009. Effects of in-season plyometric training within soccer practice on explosive actions of young players. Journal of Strength and Conditioning Research, 23(9): 26052613.

Middleton, G., D.C. Bishop, C. Smith and T.I. Gee, 2013. The implementation of a sports-specific resistance and plyometric training programme for an elite junior badminton player. Presented at the UKSCA 2013 Annual Conference, 31.08.13 - 01.09.13, Not-Tingham University. 
Rambely, A.S., W.A.B.W. Abas and M.S. Yusof, 2005. The analysis of jumping smash in the game of badminton. In International Society of Biomechanics in Sports (Ed.). Proceedings of International Symposium on biomechanics in sports conference .Beijing: International Society of Biomechanics in Sports. pp: 671-674.

Saez-Saez de Villarreal, E., B. Requena and R.U. Newton, 2010. Does plyometric training improve strength performance? A meta-analysis. Journal of Science and Medicine in Sport, 13(2): 513-552.

Sialis, J., 2004. Innervations charakteristik und training sadaptabilität im dehnungs-verkürzungs-zyklus. Stuttgart: Dissertation.

Sturgess, S. and R.U. Newton, 2008. Design and implementation of a specific strength program for badminton. Strength and Conditioning Journal, 30(3): 33-41.

Tang, H.P., K. Abe and K. Katoh, 1995. Three-dimensional cinematographical analysis of the badminton forehand smash: Movements of the forearm and hand. In: T. Reilly, M. Hughes and A. Lees (Eds). Science and Racket Sports. London: E and FN Spon. pp: 113-118.

Tsai, C.L. and S.S. Chang, 1998. Biomechanical analysis of differences in the badminton smash and jump smash between Taiwan elite and collegiate players. XVI International Symposium on Biomechanics in Sports. pp: 259-262.

Tsai, C.L., C. Huang, D.C. Lin and S.S. Cheng, 2000. Biomechanical analysis of the upper extremity in three different badminton overhead strokes. Proceedings of XVIII International Symposium on Biomechanics in Sports. Hong Kong: The Chinese University of Hong Kong. pp: 831-834.

Witzke, K.A. and C.M. Snow, 2000. Effects of plyometric jump training on bone mass in adolescent girls. Medicine and Science in Sports and Exercise, 32(6): 1051-1057.

Wu, Y.K., Y.H. Lien, K.H. Lin, T.T. Shih, T.G. Wang and H.K. Wang, 2010. Relationships between three potentiation effects of plyometric training and performance. Scandinavian Journal of Medicine and Science in Sports, 20(1): 80-86. 